\title{
Descriptor-Free Representation of Arrays with Dependent Types
}

\author{
Kai Trojahner ${ }^{1}$ and Clemens Grelck ${ }^{2,3}$ \\ 1 Institute of Software Technology and Programming Languages \\ University of Lübeck, Germany \\ trojahner@isp.uni-luebeck.de \\ 2 Institute of Informatics \\ University of Amsterdam, Netherlands \\ c.grelck@uva.nl \\ 3 Department of Computer Science \\ University of Hertfordshire, United Kingdom \\ c.grelck@herts.ac.uk
}

\begin{abstract}
Besides element type and values, a multidimensional array is characterized by the number of axes (rank) and their respective lengths (shape vector). Both properties are essential to do bound checking and to compute linear offsets into heap memory at run time. In order to have an array's rank and shape available at any time during program execution both are typically kept in an array descriptor that is maintained at run time in addition to the array itself.

In this paper, we propose a different approach: we treat array rank and shape as first-class citizens themselves. Firstly, we use dependent types to reflect structural properties of arrays in the type system. Secondly, we annotate a program with the array explicit array properties wherever necessary. This choice not only renders implicit run time array descriptors obsolete, but exposing all rank and shape computations explicitly in intermediate code also allows us to perform extensive compile time optimisation on them. We have implemented the proposed approach in our experimental array language Qube; preliminary experimental results indicate the suitability of the proposed approach.
\end{abstract}

\section{Introduction}

Multidimensional arrays as found in ApL [1], MAtLaB [2], and SAC[3,4] are, apart from the data they contain, characterized by two properties: a rank scalar and a shape vector. An array's rank denotes the array's number of axes. Its shape vector contains the array's extent along each axis. Thus, for any given array, the length of its shape vector equals its rank.

Array ranks and shape vectors are essential for the evaluation of shapegeneric array programs: when we select the element $A_{i, j}$ from a matrix $A: \mathbb{R}^{m \times n}$ stored in row-major order, the offset into the linear memory representation of $A$ is $i * n+j$. Both extents $m$ and $n$ are necessary to check whether the indices $i$ and $j$ range within the array boundaries. In general, not even array rank is a compile 
time constant. If so, both bound checking and offset computation require to loop over the shape vector and the index vector.

To provide rank and shape vector whenever necessary, language implementations usually associate each array with its respective properties at run time. For example, one-dimensional arrays may be represented as pairs of the form $\left\langle l,\left[d_{1}, \ldots, d_{l}\right]\right\rangle$ where $l$ denotes the array's length. To the rest of compiled code, an array typically appears as an abstract data type, providing means for obtaining array length and (safely) accessing individual elements. Extending this scheme to truly multidimensional arrays yields tuples $\left\langle r,\left[s_{1}, \ldots, s_{r}\right],\left[d_{1}, \ldots, d_{p}\right]\right\rangle$ where $r$ denotes the rank and $\left[s_{1}, \ldots, s_{r}\right]$ the shape vector. As as data type invariant, $p=\Pi_{i=1}^{r} s_{i}$ must hold for all arrays.

While a descriptor-based run time representation of arrays is intuitive, the information in the descriptors is often redundant. We observe that array programs typically contain relationships between ranks, shapes, and elements of different arrays. For example, the shape-generic function add takes two arrays of arbitrary but identical shape and yields yet another array of that shape. The result contains the element-wise sum of the arguments.

add $\mathrm{a} b=$ gen $\mathrm{x}<$ (shape a) with (a. $[\mathrm{x}]+\mathrm{b} \cdot[\mathrm{x}])$;

Through the descriptors of $a$ and $b$, rank and shape are passed to the operation twice and returned once more into the calling context through yet another descriptor. Moreover, the expression shape a queries the shape vector of a: the shape vector is copied from the implicit descriptor of a into a newly allocated array whose descriptor merely contains the rank of a. As the value of shape a is used to specify the shape of the result of add, its elements are copied again into the function result's descriptor.

To eliminate such redundancies, we propose a descriptor-free evaluation scheme for multidimensional arrays. The key idea is to use dependent types to capture the array properties symbolically at compile time. We use this information to statically annotate intermediate code with terms representing array properties wherever they are required. Any operation in the intermediate code that needs an array's rank or shape vector is extended to accept this information explicitly through additional arguments. Each expression that originally evaluates to an array, may then only evaluate to a mere vector that contains the array's elements. Through this transformation, we emancipate ranks and shape vectors, which are now first-class arrays themselves.

Once all structural properties of arrays and their relationships are represented explicitly in intermediate code, they become subject to program optimisation. For example, dead code removal eliminates superfluous computations of and on structural properties. Likewise, common subexpression elimination avoids repeated computation of identical structural information. Constant folding, algebraic simplification and strength reduction contribute their share to optimise computations on structural array properties.

We have implemented the proposed descriptor-free run time representation of arrays in our functional array language Qube [5]. Qube, essentially, is an experimental offspring of the generic functional array language $\mathrm{SAC}$ [6]. Unlike SAC, 
Qube employs a type system with dependent types to provide static guarantees for the successful evaluation of array programs. Beyond rendering dynamic checks obsolete, the combination of dependent types and the descriptor-free run time representation of arrays, is a particularly fruitful one. Dependent array types allow us to express relationships between the ranks and shapes of argument and result arrays of functions explicitly. It is this information in particular that triggers optimisation both within individual function definitions and across function applications. As a consequence, we can often substantially reduce the number of explicit shape and rank values compared to the number of implicit descriptors in a conventional setting.

The remainder of this paper is organized as follows: Section 2 introduces the basic concepts of Qube by means of a core language; we assign a static semantics to the core language by defining the essential typing rules in Section 3. The dependent types are then used in Section 4 to annotate the program with rank and shape information wherever this information will be required at run time. In Section 5, we define an operational semantics for the annotated language that does not require dynamically looking up array properties. We quantify the impact of our approach by means of a custom benchmark in Section 6 . In Section 7 we describe related work in the field before we conclude the paper in Section 8.

\section{Array programming basics}

For our presentation, we define a core language that enhances the $\lambda$-calculus with essential array programming features. Figure 1 shows the syntax and an operational semantics of the core language. In addition to the usual variables, $\lambda$-abstractions and function applications, the language provides means for array creation, array inspection and some vector arithmetic. The core language employs a call-by-value evaluation regime, i.e. the evaluation of nested expressions always starts with the leftmost-innermost (closed) subexpression.

In the array programming paradigm, all values are multidimensional arrays. Each array is characterized by its rank denoting its number of axes and a shape vector that describes the length of each axis. For example, a vector [ $\left.\begin{array}{lll}1 & 2 & 3\end{array}\right]$ has rank 1 and shape [3]. In this setting, a scalar value such as 23 is also an array with rank 0 and shape [].

Array values have the form $\left[\left|q^{p}\right|\left[s^{d}\right] \mid\right]^{4}$ with $p=\operatorname{prod}\left(s^{d}\right)$. The integer vector $\left[s^{d}\right]$ is called the array descriptor. It serves as a run time representation of the shape vector. In our notation, the length $d$ of $s^{d}$ implicitly encodes the array rank. A computing machine, however, must explicitly encode $d$ in some way. The data vector $q^{p}$ contains the array elements in row-major order as a sequence of quarks. These are shapeless entities and may thus only occur inside of arrays. Quarks may be primitive values such as the integers $c$, abstractions $\lambda x . t$ mapping arrays to arrays, and tuples of arrays (not discussed in this paper). To ease the notation of scalars, we define $c \equiv[|c|[] \mid]$ and $\lambda x . t \equiv[|\lambda x . t|[] \mid]$.

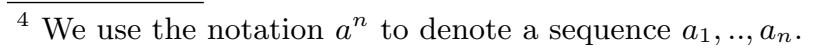


Both the data vector and the array descriptor are essential for the evaluation of array programs. E.g. an application $t t^{\prime}$ can only be reduced according to rule E-APP if $t$ is a scalar array that contains a single $\lambda$-abstraction.

The built-in functions $\operatorname{dim}(t)$ and shape $(t)$ yield the rank and shape vector of $t$ by inspecting the descriptor of their evaluated argument. Similarly, length $(t)$, if applied to a vector, yields its length as a scalar. The selection $\operatorname{sel}\left(t_{1}, t_{2}\right)$ obtains the element of $t_{1}$ that is located at the position $t_{2}$. Evaluating the selection requires that the length of the index vector $t_{2}$ equals the rank of $t_{1}$ and that $t_{2}$ denotes a position inside of $t_{1}$. The function $\iota\left(d, s^{d}, i^{d}\right)$ computes the linear offset of the index vector $i^{d}$ into the data vector of an array of rank $d$ and shape $s^{d}$.

Integer vectors have special significance in array programming as they serve as both index and shape vectors. When $t_{l}$ is a non-negative integer, $\operatorname{vec}\left(t_{l}, t\right)$ evaluates to a vector of length $t_{l}$ that contains the scalar $t$ at each index. cat $\left(t_{1}, t_{2}\right)$ appends a vector $t_{2}$ to a vector $t_{1}$. Conversely, vectors are split by the operations take and drop. For a given vector $t$ of length $m$ and an integer $t_{n}$ with $0 \leq t_{n} \leq m$, take $\left(t_{n}, t\right)$ and $\operatorname{drop}\left(t_{n}, t\right)$ yield the prefix of $t$ of length $t_{n}$ and the suffix of $t$ of length $m-n$, respectively.

Linear binary operations such as,+- , etc. may be applied to both pairs of scalars and pairs of equally sized integer vectors. Thereby, $\tilde{f}$ represents the operation denoted by the symbol $f$.

The array constructor $\left[t^{p} \mid\left[f^{d}\right]\right]$ with $\forall i . f_{i}>0, p=\operatorname{prod}\left(f^{d}\right)$, and thus $p>0$ creates a new array of frame shape $f^{d}$ by evaluating the cell expressions $t_{j}$. When all cells evaluate to arrays of identical shape $c^{r}$, the new array's descriptor is cat $\left(f^{d}, c^{r}\right)$. Its data vector is the concatenation of the cells' individual elements. For example

$$
[[|1,2|[2] \mid],[|1,2|[2] \mid] \mid[2]] \rightarrow[|1,2,3,4|[2,2] \mid]
$$

An array constructor cannot specify arrays without cells: although an array may not contain any quarks, the descriptor suffix $c^{r}$ would be unknown.

Arrays with a dynamic frame shape may be specified using the WITH-loop, an array comprehension inherited from SAC. When $t_{f_{s}}$ evaluates to a positive integer vector, an expression gen $x<t_{f s}$ with $t_{c}$ evaluates an array constructor of frame shape $t_{f s}$. The WITH-loop binds the index vector $x$ that may appear free in the cell expression $t_{c}$. Each cell is computed by instantiating $t_{c}$ such that $x$ is replaced with a vector that denotes the cell's location inside the frame. Using the WITH-loop, we can for example map a function $f$ to an arbitrarily shaped array $a$ of arguments:

gen $x<\operatorname{shape}(a)$ with $(f \operatorname{sel}(a, x))$

Although elegant, the specification is not total as a may be an empty array whose shape vector contains at least one zero. In this case, the with-loop evaluates to an ill-formed array constructor without cells.

In this section, we have specified an untyped core language for functional array programming. Each array is represented by both its elements and a descriptor, a run time data structure that describes the array's rank and shape. 


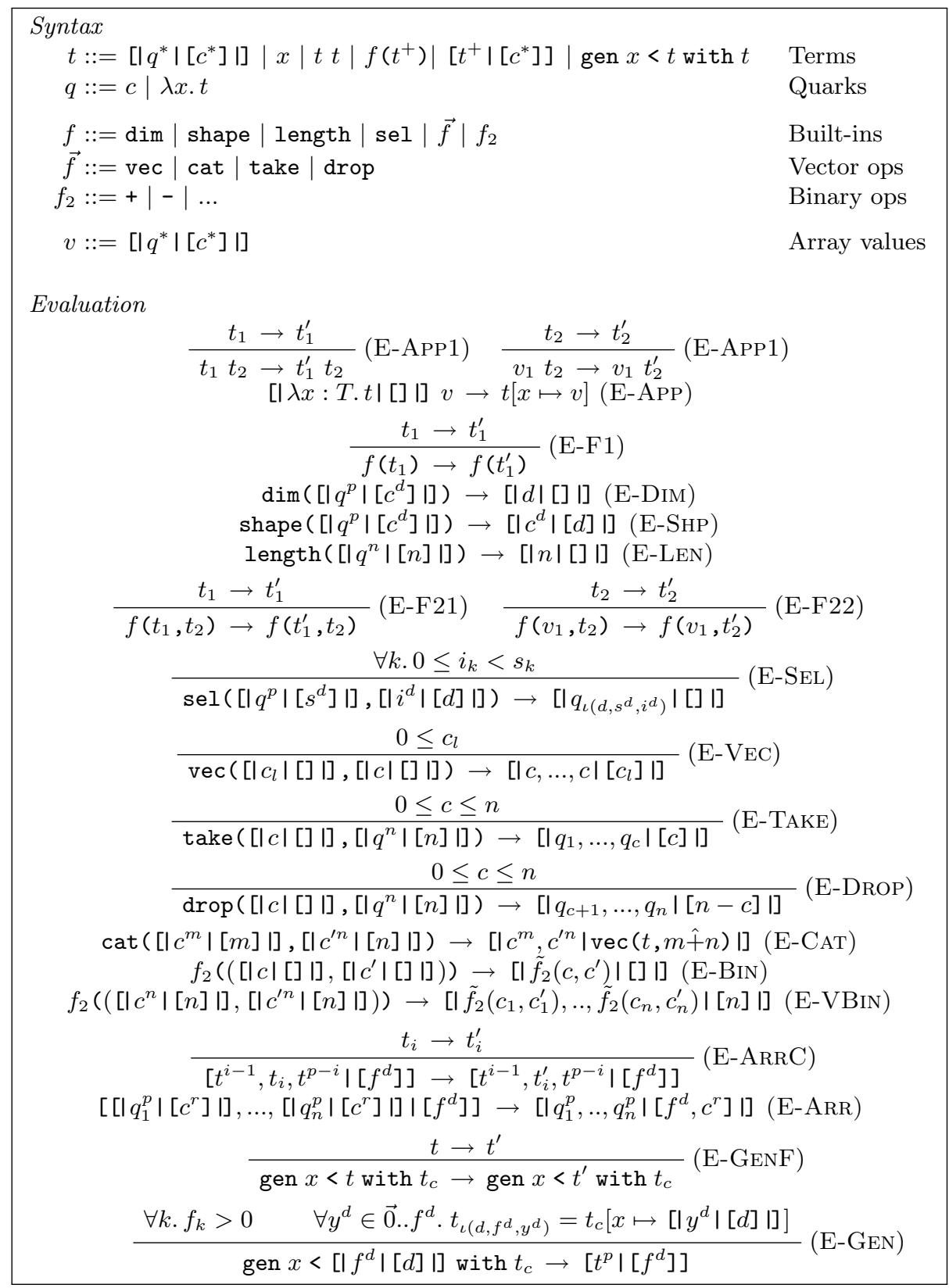

Fig. 1. A core language for functional array programming

Descriptors are in two ways essential for the evaluation of array programs: First, they are required to check whether a term satisfies the necessary preconditions 


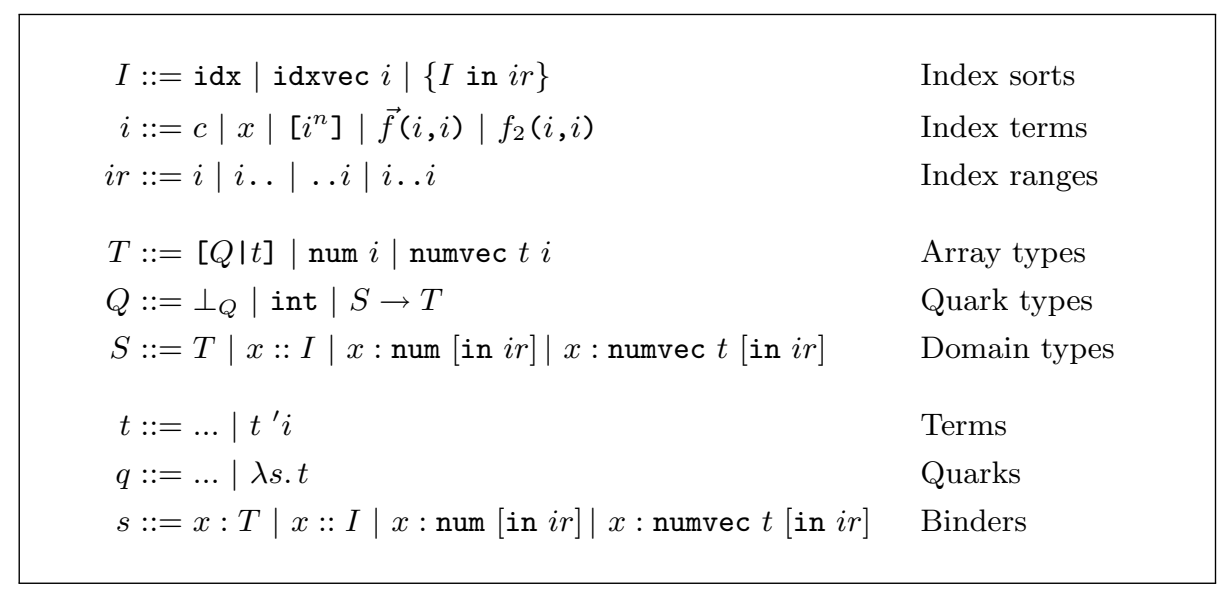

Fig. 2. Dependent array types for the core language

for further evaluation. Second, they are relevant for computing the result of an evaluation step. Nonetheless, we aim at representing arrays without descriptors.

\section{$3 \quad$ Types}

The focus of this paper lies on harnessing type information to evaluate array programs without descriptors. Therefore, our presentation of the type system concentrates on how to gather and represent the necessary information and guarantee orderly evaluation. For a more thorough treatment of the type system see $[5]$.

Our approach for eliminating run time descriptors relies on extensive use of compile time information about the arrays. We use dependent types to represent not only element type on an array but also the array's rank and shape vector. In the presence of unbounded recursion, type checking of a programming language with dependent types is undecidable [7]. To make type checking decidable, our approach resembles an indexed type system $[8,9]$ that only allows types to depend on compile time terms of a specific index language, on which constraints may be resolved statically.

We intend to annotate our programs with the rank and shape information from the array types. This rules out standard indexed types as the indices are only available at compile time. Therefore, we index our family of array types with proper language terms. However, these terms must be of a singleton type, i.e. the value of the expression describing an array's shape must be associated with a compile time index. This way, we may put run time terms into programs wherever shape and rank information is required. Yet, we may statically reason about array ranks, shape vectors, and even values by means of a constraint solver. 
Fig. 2 shows the extensions we make to the core language. Our index language consists of integer scalars of sort idx and integer vectors which belong to a member of the sort family idxvec $i$ where $i$ denotes the vector length. Index terms may be variables, integers, vectors of scalars and linear operations applied to index terms. Index vectors may be may also be formed with the structural operations vec, take, drop, and cat. Index sorts can be restricted to specific ranges using the subset notation $\{I$ in $i r\}$. A range $a . . b$ denotes all $x$ for which $a \leq x<b$. Both boundaries may be omitted, indicating $\pm \infty$ as the boundaries. Sort checking is decidable if the structural operations allow to statically decompose structured vectors into atomic vector fragments [5].

The singleton type num $i$ characterizes scalar integers whose value is denoted by the index term $i$. Similarly, the type numvec $t_{l} i$ describes specific integer vectors where $t_{l}$ is a non-negative num. More generally, $[Q \mid t]$ is the type of all arrays whose elements have quark type $Q$ and whose shape vector is characterized by the non-negative numvec $t$. By subtyping, each num $i$ is also an [int []$]$ and a numvec $t i$ is also an [int| $[t]]$.

The bottom quark type is a subtype of all quark types. It serves as a quark type for empty arrays like the empty vector [I I [0] I] which has type $\left[\perp_{Q} \mid[0]\right]$. Populated arrays may contain primitive data such as integers and functions. Regular functions of type $T \rightarrow T$ map arrays to arrays, whereas the type $x:: I \rightarrow T$ allows us to specify functions whose result type depends on an index. Additionally, functions may depend on singleton arguments: the type $x:$ num in $i r \rightarrow T$ simultaneously binds an index $x$ in the range $i r$ and an expression of type num $x$.

The basic typing rules for quarks and terms are summarized in Fig. 3. Note that through subtyping quarks and terms have multiple types.

The type rule T-VAL for non-empty array values $\left[\left|q^{p}\right|\left[s^{d}\right] \mid\right]$ checks that each quark $q_{i}$ has the same quark type $Q$. For an empty array value without quarks, no precise quark type can be determined. For this reason, rule T-VALE assigns it the bottom quark type $\perp_{Q}$, which is a subtype of any quark type. In addition to their array types, constant integer scalars and vectors also have more specific constant singleton types according to the rules T-Num, T-NumVEC.

The rules T-APP, T-IAPP, T-NAPP, and T-VAPP ensure that only scalar arrays of (dependent) functions can be applied to suitable arguments. The result type of a dependent function application is obtained by replacing occurrences of the bound variable in the co-domain with the concrete argument.

Typing of the array specific operations is shown in Fig. 4. The dim and shape primitives can be applied to arbitrary arrays and yield singleton types. length is only applicable to singleton vectors and yields a scalar singleton. The typing rule T-SEL statically enforces all the necessary preconditions of the selection: the selection vector must be a singleton vector with appropriate length that ranges within the boundaries of the array selected into. A (valid) selection always yields a scalar array but never a singleton.

The vector operations vec, cat, take, and drop always require appropriate singleton arguments and yield a singleton vector whose index is formed in the same way. Three rules are used to type applications of binary operations: They 
may be applied to integer scalars (T-BIN), yielding another of the same element type and shape. More interestingly, when applied to (compatible) singletons ( $\mathrm{T}$ BINS, T-BINV), the result is also a singleton whose index is characterized by the application of the operation to the original singletons' indices.

An array constructor with frame shape $\left[c^{n}\right]$ is well-typed if all cells have the same quark type $Q$ and the same shape $t_{s}$. The new array then has type $\left[Q \mid \operatorname{cat}\left(\left[c^{n}\right], t_{s}\right)\right]$. In the special case where all cells of a vector are singleton

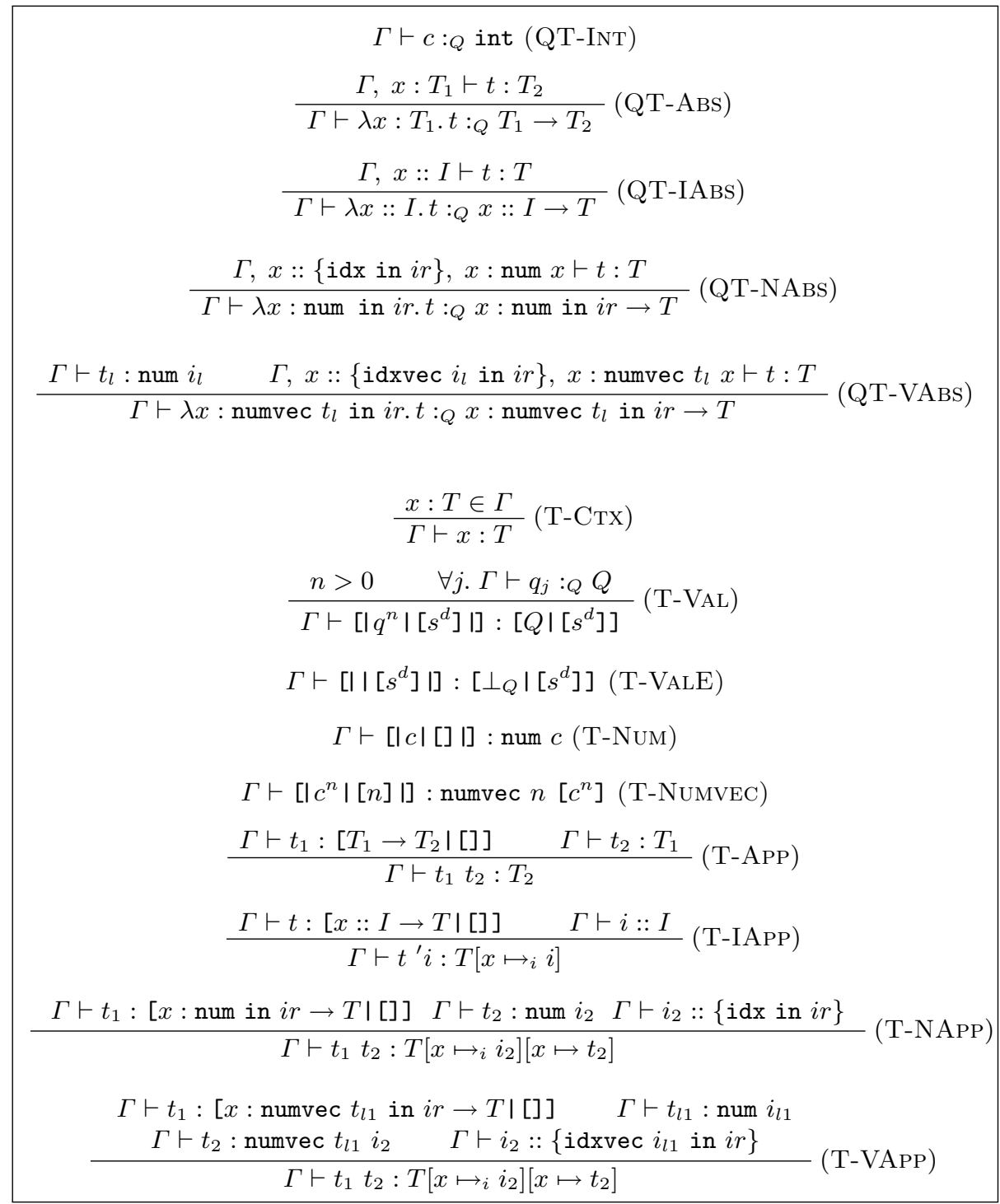

Fig. 3. Basic typing rules 


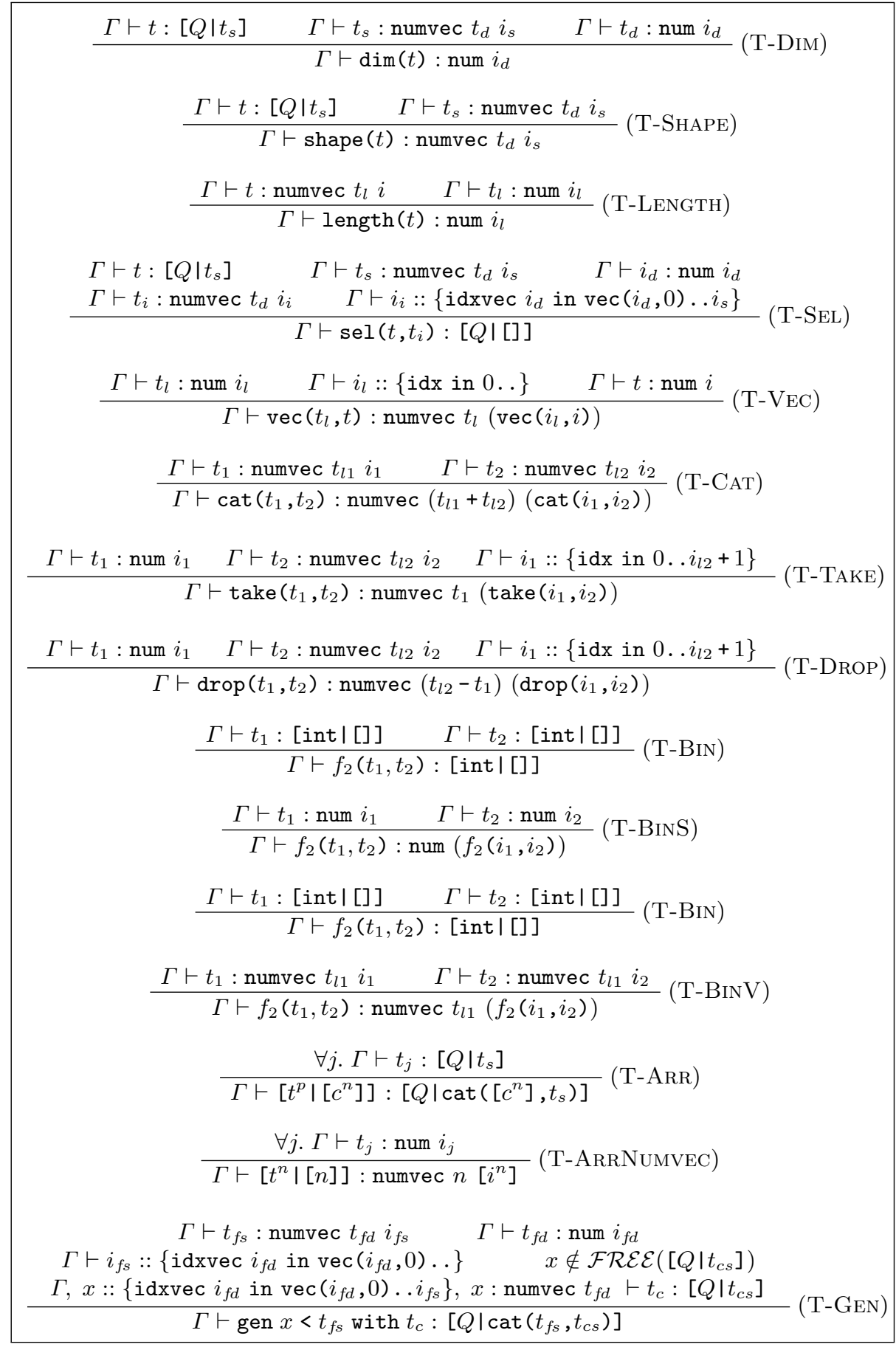

Fig. 4. Typing rules for array specific language elements 
scalars, rule T-ARRNUMVEC gives the array the appropriate singleton vector type. The typing rule T-GEN for the WITH-loop gen $x<t_{f s}$ with $t_{c}$ verifies that the frame shape $t_{f s}$ is a non-negative vector. For checking the cell expression $t_{c}$, the identifier $x$ is bound to both a vector sort that ranges between zero and the frame shape and a singleton vector with exactly that value. If the cell expression then has type $\left[Q \mid t_{c s}\right]$, then the WITH-loop has type $\left[Q \mid\right.$ cat $\left.\left(t_{f s}, t_{c s}\right)\right]$. The type rule allows WITH-loops with empty frame shapes as these will be enabled by the modified semantics in Section 6 .

Type checking rules out all programs that may not satisfy the constraints on ranks, shape vectors, and array values inherent to array programs. Program evaluation may thus entirely dispense with dynamic checks of these constraints. Still, array descriptors are required whenever array properties determine the evaluation result.

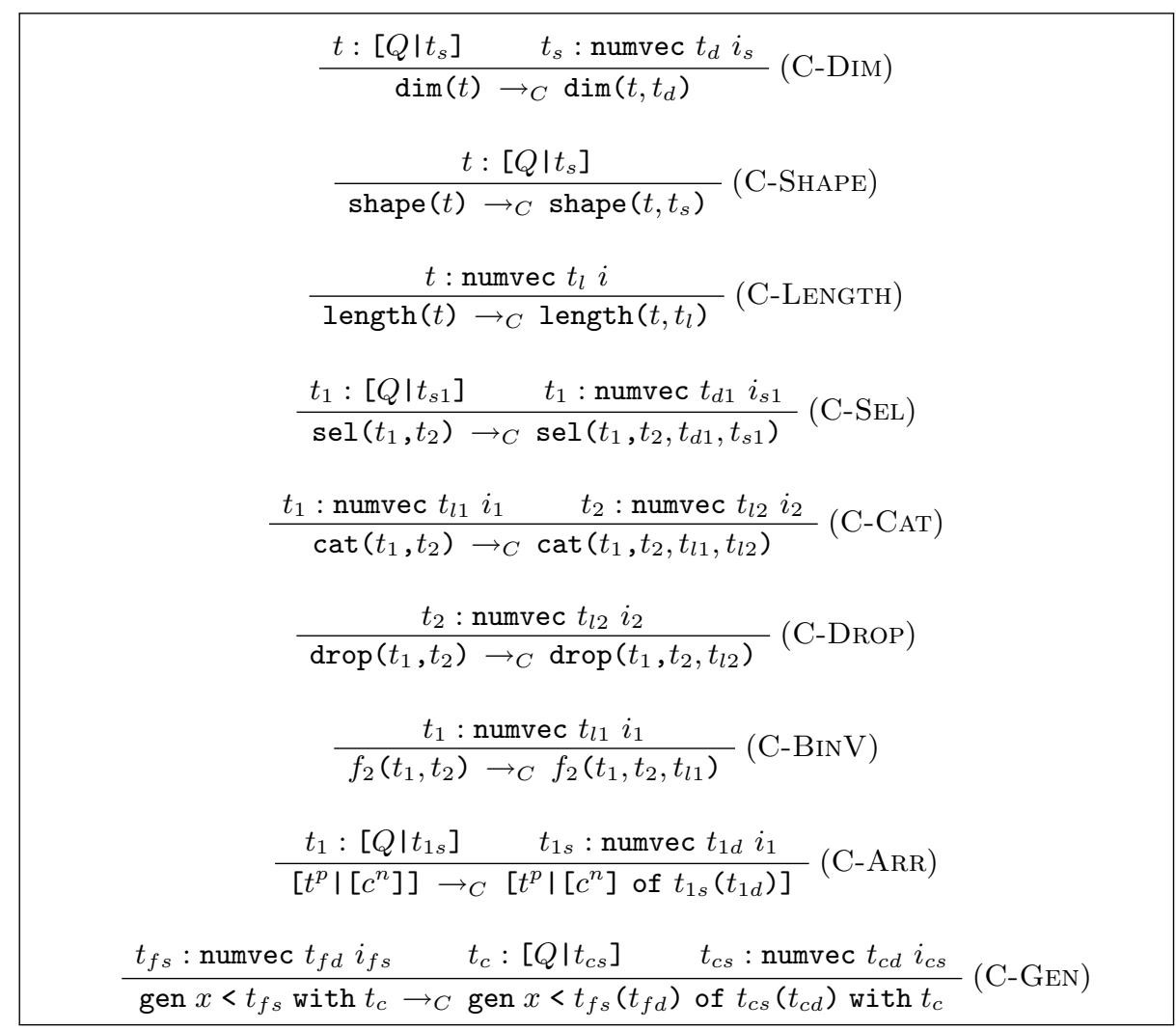

Fig. 5. Static annotation of the required array properties 


\section{Array properties on demand}

By construction, the type of an array $\left[Q \mid t_{s}\right]$ encodes both the array's quark type $Q$ and its shape vector $t_{s}$. As $t_{s}$ must be a numvec $t_{d}$, its length $t_{d}$ also denotes the array's rank. Thus, both rank and shape vector are symbolically known at compile time. We annotate the program with these terms wherever array properties will be required for program evaluation. In Section 5 we will define an operational semantics that takes the annotations into account. Ultimately, this allows us to entirely eliminate array descriptors.

Fig. 5 shows the relation $\rightarrow_{C}$ for statically annotating array programs with the array properties that will be required at run time. The rules are applied exhaustively throughout the program. By virtue of type checking, the premises hold for every well-typed program. They are only required to match the desired type patterns.

The evaluation of function applications and scalar operations does not require any array properties.

The operations $\operatorname{dim}(t)$, shape $(t)$, and length $(t)$ yield array properties for which terms may be directly derived from the type of $t$. Instead of replacing the entire expressions, we merely extend the argument lists in order to maintain the call-by-value semantics. The selection sel $\left(t, t_{i v}\right)$ requires both the rank $t_{d}$ and the shape vector $t_{s}$ of $t$ to compute the offset into the data vector of $t$ through $\iota\left(t_{d}, t_{s}, t_{i v}\right)$.

A vector construction vec $\left(t_{l}, t\right)$ and a vector prefix take $\left(t_{l}, t\right)$ don't require additional information at run time as the result lengths are given by the argument $t_{l}$ itself. However, computing the suffix of a vector with $\operatorname{drop}\left(t_{d}, t\right)$ also requires the length of $t$. The concatenation cat $\left(t_{1}, t_{2}\right)$ depends on the lengths of both argument vectors. In contrast, the arguments of well-typed linear vector arithmetic operations must have the same length; we thus only annotate the length of the first vector.

The size of an array defined by an array constructor $\left[t^{p} \mid\left[c^{d}\right]\right]$ depends on both the static frame shape $\left[c^{d}\right]$ and the size of the array cells. The size of an array cell is the product of its shape vector. Since all cells must have the same rank and shape, we annotate the constructor with the first cell's rank and shape vector. For the same reason, a wiTH-loop gen $x<t_{s}$ with $t_{c}$ is annotated rank and shape vector of the cell expression $t_{c}$. As the WITH-loop's frame shape $t_{s}$ is a vector expression, we also annotate its length.

\section{Evaluation of annotated array programs}

In this section, we redefine the evaluation relation $\rightarrow$ for well-typed programs such that it uses the property annotations instead of dynamically accessing array descriptors. No dynamic checks are performed as potential run time errors have been ruled out by means of type checking. Fig. 6 shows the modified semantics. We underline the descriptor in an array value $\left[\left|q^{p}\right|\left[s^{d}\right] \mid\right]$ to indicate that it is a mere compile time attribute that is required for type checking. At run time however, no descriptor will be built. 


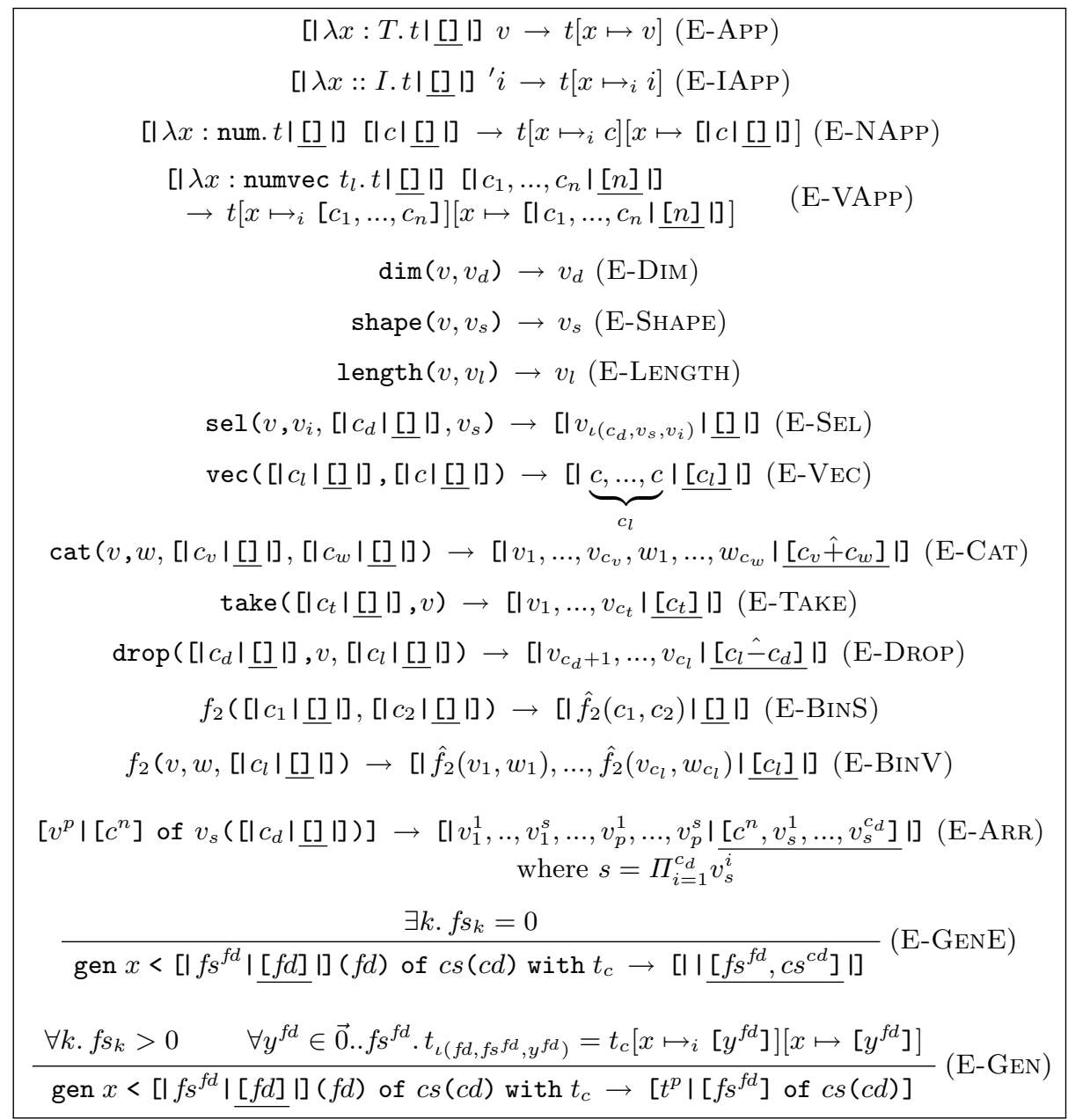

Fig. 6. Descriptor-free evaluation of well-typed programs with property annotations

Evaluation of function applications does not require any array properties. The array inspection operations dim, shape, and length have been annotated with terms denoting the sought array properties. They evaluate directly to their second argument, ignoring the inspected array.

The selection blindly fetches the indexed element from the data vector as out-of-bounds accesses cannot occur and the relevant information for computing the linear offset is available. The modified evaluation of the vector operations is similarly straightforward.

Annotated array constructors evaluate to an array value whose size is determined by the static frame shape and the dynamic cell shape vector $c s$ of length 
$c d$. All cells will evaluate to arrays of that shape. Thus, the new data vector is formed by copying prod(cs) quarks from each cell array.

The evaluation rule E-GEN for the WITH-loop is adjusted to the typed, annotated setting. The substitution of the index vector in the cell expression now takes type indices into account and the annotated cell shape is transcribed to the resulting array constructor. More interestingly, the additional evaluation rule E-GENE specifies that annotated WITH-loops with empty frame shape are evaluated to empty array values with an appropriate (compile time) descriptor. Hence, we may use WITH-loops to define arbitrary shape-generic operations even if the frame shape is empty.

\section{Experimental evaluation}

We have implemented the proposed descriptor-free evaluation of array programs in our functional array language Qube. Essentially, Qube is an experimental offspring of SAC [6]. Its notation is similar to that of HASKELL. We use Qube to explore how dependent array types can be harnessed to compile verified shapegeneric programs into efficient, data-parallel code.

\subsection{Implementation}

Our language implementation compiles Qube programs into C code which, in turn, gets compiled into native executables by a $\mathrm{C}$ compiler of the user's choice. The implementation is currently limited to first order functions. For efficiency, we use two array representations: scalars are held on the $\mathrm{C}$ run time stack or even in registers whereas the data vectors of non-scalar arrays are allocated on the heap. Automatic memory management relies on the Boehm-Dehmers-Weiser conservative garbage collector [10]. In the future, we would like to replace it with a memory management scheme based on reference counting, since this would allow us to perform destructive array updates even in our context of immutable arrays [11]. Our compiler performs basic program optimisations: dead code elimination, constant folding, common subexpression elimination. Furthermore, it avoids the costly creation of WITH-loop index vectors and index computations as described in [12].

The measurements were performed on an Apple Macmini with an Intel Core Duo 2 clocked at $2 \mathrm{GHz}$ with $4 \mathrm{MB}$ L2 Cache and $2 \mathrm{~GB}$ of RAM. We use GCC version 4.2 as a back-end compiler.

\subsection{A custom benchmark}

Array descriptors add an overhead to each array. To quantify the precise effect of descriptor-free evaluation, we use a custom micro benchmark.

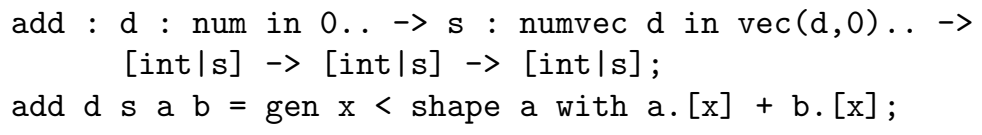


The rank-generic function add adds the elements of two equally shaped integer arrays and returns the result. Fig. 7 shows the compiled Qube program. It calculates the size of the new array, allocated memory and computes the result with virtually no overhead. To simulate the behaviour outlined in Section 2, we annotated the $\mathrm{C}$ program with array descriptors. The modified program thus keeps separate rank scalars and shape vectors for each array. It also has to compute shape a by creating a new array that mirrors the shape vector of a. In both programs, scalar arrays are represented as primitive $\mathrm{C}$ values. Moreover, both neither perform dynamic constraint checks such as array bounds checks nor index vector computations.

We use add to iterate the addition of two matrices of varying size $N \times N$ such that the overall number of scalar additions is roughly $2 * 10^{9}$. Fig. 8 shows the run time results. The descriptor-free program performs substantially better than the descriptor-based program for small arrays sizes. For matrices larger than $32 \times 32$, the overhead of descriptor maintenance becomes less significant and virtually vanishes for arrays larger than $1024 \times 1024$. The running times are dominated by memory management: a modified version of the descriptor-free program that destructively updates the first argument performs clearly superior.

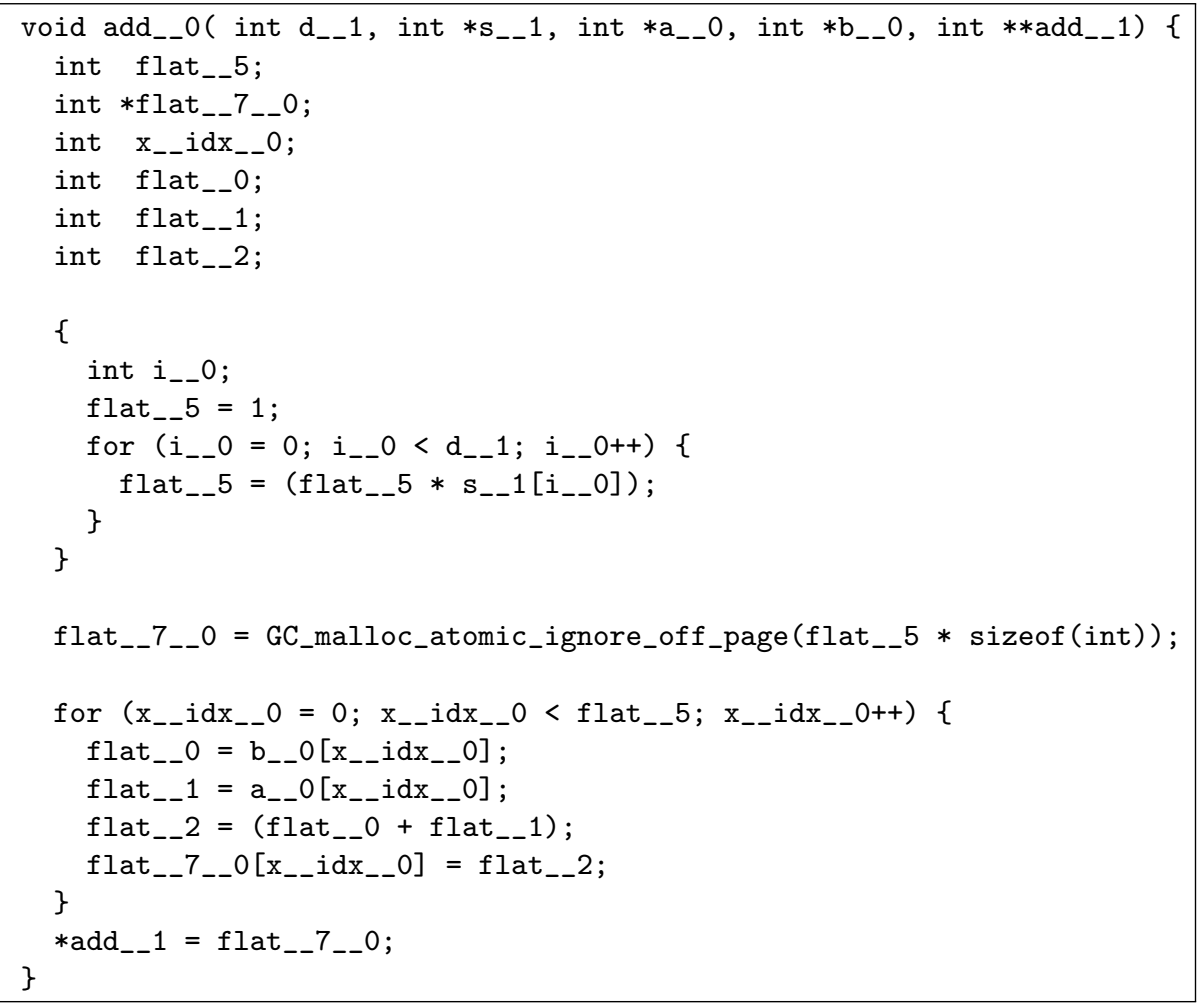

Fig. 7. The compiled C program for shape-generic array addition 


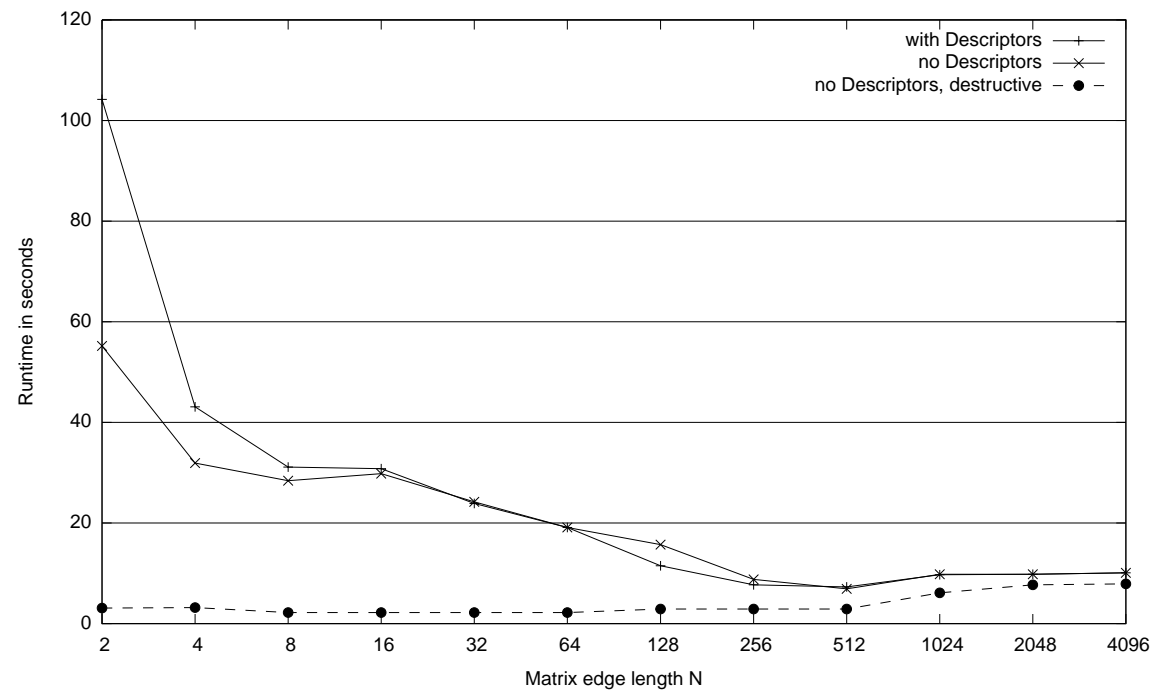

Fig. 8. Run time measurements for $2^{31} \approx 2 * 10^{9}$ integer additions organized as elementwise array additions for varying array sizes.

Execution times increase for all tested programs once the argument and result arrays become too big for the processor's L2 Cache.

\section{Related work}

Many array languages like MatLab [2], Apl [13,1], J [14] or Nial [15] are interpreted and untyped. These languages are known for offering a plethora of well optimised operators for each array operation supported by the language. They rely on the ability to dynamically access array properties via some form of descriptor.

Static knowledge about the array properties is crucial for compiling array programs into efficient executables. For example in FISH [16], each function $f$ is accompanied by a shape function \#f which maps the shape of the argument to the shape of the result. Shape inference proceeds by first inlining all functions and then statically evaluating all shape functions. FISH rejects all programs that contain non-constant array shapes.

The array language $\mathrm{SAC}$ uses different types [3] for arrays of statically known shape (e.g. int $[10,10])$, arrays of statically known rank (but unknown shape) (e.g. int [.,.]), and arrays of unknown rank and shape (e.g. int [*]). A compilation scheme for shape-generic SAC programs has been devised that takes the array types into account to avoid creating run time descriptors whenever possible [17]. To improve the static shape information, the SAC compiler uses a combination of partial evaluation and function specialisation [18]. Recently, we proposed symbolic array attributes as a uniform scheme to infer and represent 
structural information in shape-generic array programs such that it may be used by optimisations [19].

Light-weight dependently typed languages such as Xi and Pfenning's DML [9], Xi's Applied Type System [20], and Zenger's indexed types [8] are also related to our approach. These allow term-indexing into type families only by means of compile time index terms. As such, these indices cannot replace run time descriptors. In contrast, AGDA [21] and EpIGRAm [22] support types that depend on arbitrary terms but offer no particular support for shape-generic array programming. In his thesis [25], Brady showed how the rich type information in EPIGRAM programs may be used for the compilation into efficient G-machine code. More recently, Swierstra and Altenkirch proposed an AGDA library for specifying locality aware algorithms over distributed arrays [24]. The YNOT project aims at combining dependently typed programming systems with effects [23].

\section{Conclusion and future work}

In this paper, we have proposed a new method for evaluating array programs without dynamically tagging arrays with a descriptor. Dependent types allow us to represent an expression's rank and shape in the type system. We use this information to statically annotate an intermediate program with array properties wherever these are required for evaluation. Evaluation rules that take the annotations into account entirely dispense with the need for array descriptors. In addition, type checking renders dynamic constraint checks such as array bounds checks superfluous. The compiled programs thus contain very little overhead. Preliminary run time measurements indicate that descriptor-free evaluation can positively influence run time performance, especially when a program deals with many small arrays.

In the future, we would like to further develop the Qube compiler and implement more complex programs to demonstrate our approach. The existing results indicate that a memory management scheme based on reference counting will benefit the evaluation efficiency as it would allow us to often perform array updates destructively. Our general aim is to use the information present in the array types to aggressively optimise array programs. Ultimately, this will allow us to generate more efficient array programs both for sequential and parallel execution.

Acknowledgments We thank Florian Büther and Markus Weigel for contributing to the Qube project. Moreover, we also thank Walter Dosch for some interesting discussions on the topic. We also thank the anonymous reviewers for their helpful comments on the draft version of this paper.

\section{References}

1. Falkoff, A., Iverson, K.: The Design of APL. IBM Journal of Research and Development 17(4) (1973) 324-334 
2. Moler, C., Little, J., Bangert, S.: Pro-Matlab User's Guide. The MathWorks, Cochituate Place, 24 Prime Park Way, Natick, MA, USA (1987)

3. Scholz, S.B.: Single Assignment C - Efficient Support for High-Level Array Operations in a Functional Setting. Journal of Functional Programming 13(6) (2003) $1005-1059$

4. Grelck, C.: Shared Memory Multiprocessor Support for Functional Array Processing in SAC. Journal of Functional Programming 15(3) (2005) 353-401

5. Trojahner, K., Grelck, C.: Dependently Typed Array Programs Don’t Go Wrong. Technical Report A-08-06, Schriftenreihe der Institute für Informatik/Mathematik, University of Lübeck, Lübeck, Germany (2008)

6. Grelck, C., Scholz, S.B.: SAC: A Functional Array Language for Efficient Multithreaded Execution. International Journal of Parallel Programming 34(4) (2006) 383-427

7. Barthe, G., Coquand, T.: An introduction to dependent type theory. In: Applied Semantics, International Summer School, APPSEM 2000, Caminha, Portugal, September 9-15, 2000, Advanced Lectures. Volume 2395 of Lecture Notes in Computer Science., Springer-Verlag Berlin Heidelberg New York (2002) 1-41

8. Zenger, C.: Indexed Types. Theorectical Computer Science 187(1-2) (1997) 147165

9. Xi, H., Pfenning, F.: Eliminating array bound checking through dependent types. In: Proceedings of ACM SIGPLAN Conference on Programming Language Design and Implementation, Montreal (1998) 249-257

10. Boehm, H., Demers, A., Shenker, S.: Mostly parallel garbage collection. In: Proceedings of the ACM SIGPLAN '91 Conference on Programming Languages Design and Implementation. Volume 26 of ACM SIGPLAN Notices., ACM (1991) 157-164

11. Grelck, C., Trojahner, K.: Implicit Memory Management for SAC. In Grelck, C., Huch, F., eds.: Proceedings of the 16th International Workshop on Implementation and Application of Functional Languages, IFL 2004, Lübeck, Germany, September 8-10, 2004. Volume 0408 of Technical Report., University of Kiel (2004) 335-348

12. Bernecky, R., Herhut, S., Scholz, S.B., Trojahner, K., Grelck, C., Shafarenko, A.: Index Vector Elimination: Making Index Vectors Affordable. In Horváth, Z., Zsók, V., eds.: Implementation and Application of Functional Languages, 18th International Symposium (IFL'06). Budapest, Hungary, September 4-6, 2006, Revised Selected Papers. Volume 4449 of Lecture Notes in Computer Science., Springer Verlag, Berlin, Heidelberg, Germany (2007) 19-36

13. Iverson, K.: A Programming Language. John Wiley, New York City, New York, USA (1962)

14. Iverson, K.: J Introduction and Dictionary. Iverson Software Inc., Toronto, Canada. (1995)

15. Jenkins, M.: Q'Nial: A Portable Interpreter for the Nested Interactive Array Language Nial. Software Practice and Experience 19(2) (1989) 111-126

16. Jay, C., Steckler, P.: The Functional Imperative: Shape! In Hankin, C., ed.: Proceedings of the 7th European Symposium on Programming (ESOP'98), part of the Joint European Conference on Theory and Practice of Software (ETAPS'98), Lisbon, Portugal. Volume 1381 of Lecture Notes in Computer Science., Springer Verlag, Berlin, Germany (1998) 139-53

17. Kreye, D.: A Compilation Scheme for a Hierarchy of Array Types. In Arts, T., Mohnen, M., eds.: Implementation of Functional Languages, 13th International Workshop, IFL 2001, Stockholm, Sweden, September 24-26, 2001, Selected Papers. Volume 2312 of Lecture Notes in Computer Science., Springer Verlag, Berlin, Germany (2002) 18-35 
18. Grelck, C., Scholz, S.B., Shafarenko, A.: A Binding Scope Analysis for Generic Programs on Arrays. In Butterfield, A., ed.: Implementation and Application of Functional Languages, 17th International Workshop (IFL'05). Dublin, Ireland, September 19-21, 2005, Revised Selected Papers. Volume 4015 of Lecture Notes in Computer Science., Springer-Verlag, Berlin, Heidelberg, New York (2006) 212-230

19. Trojahner, K., Grelck, C., Scholz, S.B.: On optimising shape-generic array programs using symbolic structural information. In Horváth, Z., Zsók, V., eds.: Implementation and Application of Functional Languages, 18th International Symposium (IFL'06). Budapest, Hungary, September 4-6, 2006, Revised Selected Papers. Volume 4449 of Lecture Notes in Computer Science., Springer Verlag, Berlin, Heidelberg, Germany (2007)

20. Xi, H.: Applied Type System (extended abstract). In Baradi, S., Coppo, M., Damiani, F., eds.: Types for Proofs and Programs, Third International Workshop, TYPES 2003, Torino, Italy, April 30 - May 4, 2003, Revised Selected Papers. Volume 3085 of Lecture Notes in Computer Science., Springer Verlag, Berlin, Germany (2004) 394-408

21. Norell, U.: Towards a practical programming language based on dependent type theory. PhD thesis. PhD thesis, Chalmers University of Technology, Göteborg, Sweden (2007)

22. McBride, C.: Epigram: Practical programming with dependent types. In: Advanced Functional Programming. (2004) 130-170

23. Nanevski, A., Morrisett, G., Birkedal, L.: Polymorphism and Separation in Hoare Type Theory. In: International Conference on Functional Programming, New York, NY, USA, ACM Press (2006) 62-73

24. Swierstra, W., Altenkirch, T.: Dependent types for distributed arrays. In Achten, P., Koopman, P., Morazán, M., eds.: Trends in Functional Programming. Volume 9., Intellect Books (2008)

25. Brady, E.: Practical Implementation of a Dependently Typed Functional Programming Language. PhD thesis, Department of Computer Science, University of Durham, Durham, UK (2005) 